\title{
Effect of Cardiac Rehabilitation on Myocardial Perfusion Reserve in Postinfarction Patients
}

\author{
Bai-Chin Lee, MD ${ }^{\mathrm{a}, \mathrm{b}}$, Ssu-Yuan Chen, MD, PhD ${ }^{\mathrm{c}}$, Hsiu-Ching Hsu, PhD ${ }^{\mathrm{a}}$, \\ Mao-Yuan Marine Su, MS ${ }^{\mathrm{d}}$, Yen-Wen Wu, MD ${ }^{\mathrm{a}, \mathrm{b}}$, Kuo-Liong Chien, MD, PhD ${ }^{\mathrm{a}, \mathrm{e}}$, \\ Wen-Yih Issac Tseng, MD, PhD ${ }^{\mathrm{f}, \mathrm{g}}$, Ming-Fong Chen, $\mathrm{MD}, \mathrm{PhD}^{\mathrm{a}, *}$, and Yuan-Teh Lee, MD, PhD ${ }^{\mathrm{a}}$
}

Cardiac rehabilitation is believed to increase myocardial perfusion reserve (MPR), but this has not been adequately studied because of poor delineation of infarcted myocardium in previous studies. The purpose of this study was to determine the effect of cardiac rehabilitation on MPR in the remote and infarcted myocardium with contrast-enhanced magnetic resonance imaging; 39 postinfarction patients were recruited for this study and randomly assigned to a training group $(n=20)$ or a nontraining group $(n=19)$. Those in the training group participated in a 3-month rehabilitation training program at an exercise intensity of $55 \%$ to $70 \%$ of peak oxygen uptake $\left(\mathrm{VO}_{2}\right)$; those in the nontraining group continued their usual lifestyle. Nineteen age-, weight-, and height-matched subjects without cardiovascular risk factors were selected as healthy controls. After myocardial infarction, a reduction in perfusion reserve was seen not only in the infarcted myocardium, but also in the remote myocardium. In the training group, exercise capacity increased by $15 \%(\mathrm{p}<0.01)$, to the same level as in healthy controls. The post-training MPR increased in both remote $(30 \%$, $\mathrm{p}<0.01)$ and infarcted myocardium $(25 \%, \mathrm{p}<0.05)$ and reached the same level as in healthy controls. The change in exercise capacity correlated with the change in MPR in the remote myocardium $\left(\mathrm{r}=\mathbf{0 . 5 5}, \mathrm{p}<0.001\right.$ for peak $\left.\mathrm{VO}_{2}\right)$. In the nontraining group, exercise capacity and MPR were unchanged. In conclusion, cardiac rehabilitation improves perfusion reserve in both infarcted and remote myocardium, with a parallel increase in exercise capacity. (C) 2008 Elsevier Inc. All rights reserved. (Am J Cardiol 2008;101:1395-1402)

Cardiac magnetic resonance imaging (MRI) is an excellent diagnostic tool for serial assessment of changes in left ventricular (LV) structure and function, infarct location and size, and myocardial perfusion reserve (MPR). ${ }^{1,2}$ The ability of cardiac MRI to assess concurrently and with high spatial resolution the extent of scar tissue in the myocardium and perfusion is 1 of the major strengths of this technique. The effect of cardiac rehabilitation on myocardial perfusion in patients with myocardial infarction (MI) has not been studied using this technique. The primary aim of the present study was to investigate whether cardiac rehabilitation influences perfusion differently in the infarcted and remote myocardium. The secondary aim was to assess the relation between MPR and exercise capacity.

\footnotetext{
aDepartment of Internal Medicine, National Taiwan University Hospital; ${ }^{\mathrm{b}}$ Graduate Institute of Clinical Medicine, College of Medicine, National Taiwan University; ${ }^{\circ}$ Department of Physical Medicine and Rehabilitation, National Taiwan University Hospital; ${ }^{\mathrm{d}}$ Institute of Biomedical Engineering, National Yang-Ming University; ${ }^{\mathrm{e}}$ Institute of Preventive Medicine, College of Public Health, National Taiwan University; ${ }^{\mathrm{f}}$ Department of Medical Imaging, National Taiwan University Hospital; ${ }^{\mathrm{g}}$ Center for Optoelectronic Biomedicine, College of Medicine, National Taiwan University, Taipei, Taiwan. Manuscript received September 19, 2007; revised manuscript received and accepted January 3, 2008.

The study was supported by grant NTUH094-000190 from National Taiwan University Hospital, Taipei, Taiwan.

*Corresponding author: Tel: 886-2-2312-3456, x5059; fax: 886-23322-3937.

E-mail address: mfchen@ntu.edu.tw (M-F. Chen).
}

\section{Methods}

Study design: This prospective randomized controlled study was performed at the National Taiwan University Hospital between August 2004 and December 2005. Men aged $\leq 65$ years old with a history of MI for at least 3 months before screening were eligible. The inclusion criteria were a successful procedural outcome after primary stenting during the initial MI treatment, a clinically stable course for at least 3 months after discharge, and no evidence of myocardial ischemia on initial and follow-up exercise testing. A successful procedural outcome was a patent infarct-related artery with residual stenosis $\leq 30 \%$ of the vessel diameter (without major adverse cardiac and cerebrovascular events in hospital). The exclusion criteria were effort angina, atrial fibrillation, sustained ventricular arrhythmia, New York Heart Association functional class IV symptoms, exercise-limiting diseases, severe pulmonary or renal disease, an implanted pacemaker, or claustrophobia.

At the baseline and 3-month follow-up assessments, all patients underwent a functional evaluation, which included clinical evaluation, exercise testing, and cardiac MRI. After the initial evaluation, patients were randomly assigned to the training group, which underwent a 3-month cardiac rehabilitation program, or the nontraining group, in which patients continued their usual lifestyle. Both groups were receiving stable, optimal pharmacologic treatment supervised by their physicians. The study complies with the Declaration of Helsinki. The study protocol was approved by the Ethics Committee of the National Taiwan University 


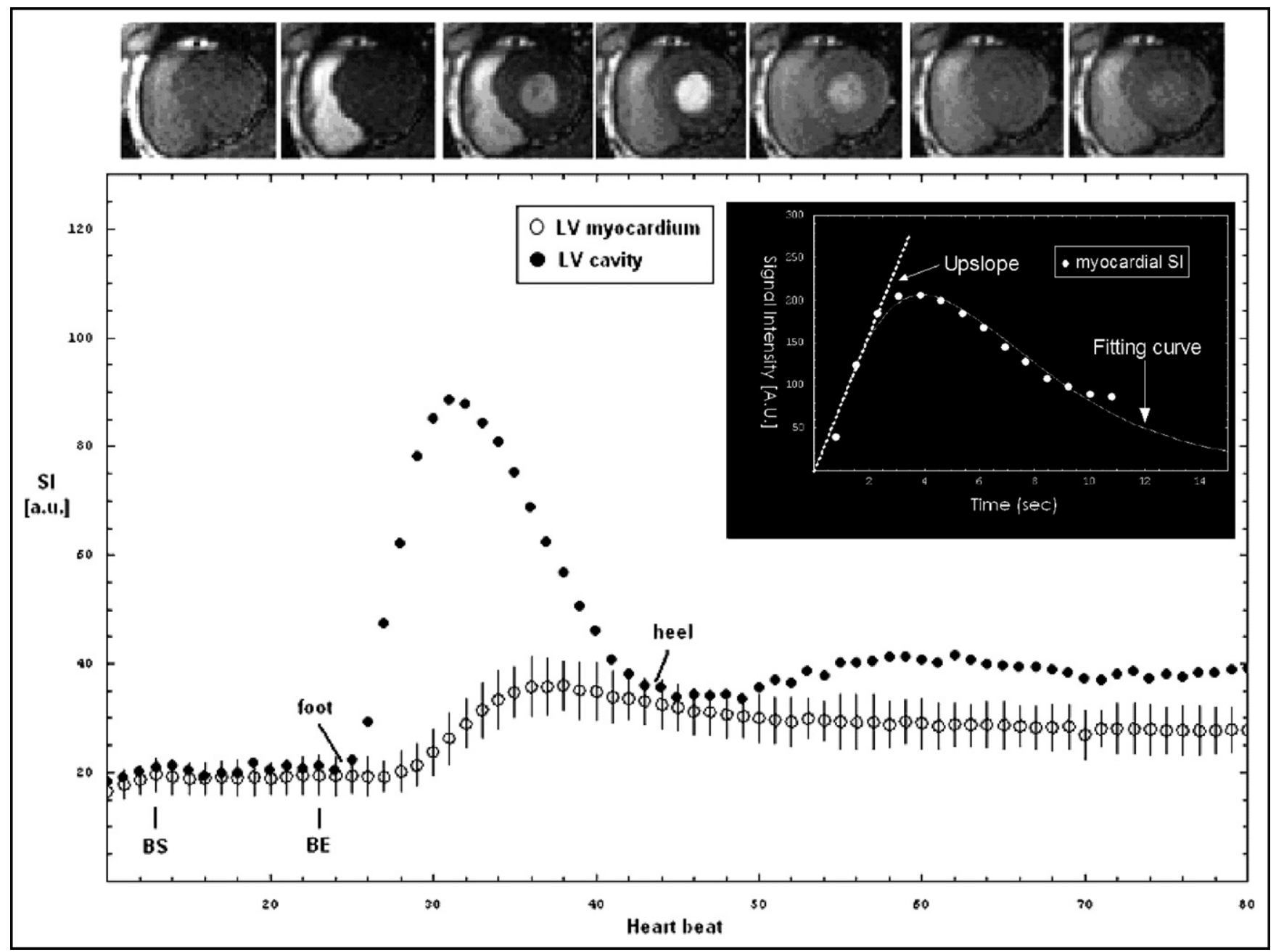

Figure 1. First-pass, contrast-enhanced myocardial perfusion images acquired for 80 heart beats in the left ventricle. Short-axis views were obtained after intravenous administration of gadodiamide (upper panels). Curves of signal intensity (SI) in arbitrary units (a.u., A.U.) in the LV cavity (closed circles) and myocardium (open circles) in a healthy subject (bottom panel). The bars represent the SDs of the means. The time window for the first pass was defined as the time of the arrival of contrast material (foot) to the time before recirculation begins (heel). The baseline SI was determined by averaging the SI from base start (BS) to base end (BE). A $\gamma$-variate function was used to smooth fit the corrected SI data, and the maximal upslope (Upslope) was calculated from the first derivative of the SI curve during the initial ascent of the first pass in each myocardial segment (inset).

Hospital, and all patients provided signed informed consent before randomization.

Study patients: Of 91 patients who were screened for possible enrollment, 37 refused to participate and 15 did not meet the inclusion criteria because of exertional angina $(\mathrm{n}=$ $3)$, sustained ventricular arrhythmias $(n=3)$, or exerciselimiting diseases $(n=9)$. The remaining 39 patients were enrolled. After completing the initial evaluation, they were randomly assigned to the 3 -month training program $(\mathrm{n}=$ $20)$ or to the the nontraining group $(n=19)$. For comparison of exercise capacity and myocardial perfusion, 19 age-, weight-, and height-matched subjects without cardiovascular risk factors were selected as healthy controls.

\section{Cardiac rehabilitation}

EXERCISE TESTING: Symptom-limited, graded exercise testing was carried out using a cycle ergometer (Ergotests, Erich Jaeger, Würzburg, Germany). ${ }^{3}$ Continuous electrocardiographic monitoring and analysis of expired gases were performed during exercise testing. The workload was 10 watts (W) for the first 3 minutes (familiarization period) and was increased by $10 \mathrm{~W}$ increments every minute. The pedaling cadence was maintained between 50 and 70 revolutions/min. Breath-by-breath analysis of the expired gas was performed using an automated system (System 2000, Medical Graphics Corporation, St Paul, Minnesota). The mean value of the peak oxygen uptake $\left(\mathrm{VO}_{2}\right)$ was determined from the final 20 seconds of the test and was expressed as $\mathrm{ml} / \mathrm{kg} / \mathrm{min}$.

TRAINING PROTOCOL: Exercise sessions were performed 3 times a week for 12 weeks at $55 \%$ to $70 \%$ of the peak $\mathrm{VO}_{2}$ measured in the initial exercise test and a perceived exertion rating of 12 to 13 (fairly light to somewhat hard) on the Borg scale. ${ }^{4}$ Each session consisted of a 5-minute warm-up period, a 20-minute bicycle-ergometer exercise, and a 5-minute cool-down period. The exercise session was supervised by a physical therapist, and heart rate and blood pressure were monitored during exercise. 
Patients in the nontraining group received educational support and continued their medications, but had no cardiac rehabilitation during the study period.

\section{Outcome measures}

CARDIAC MRI PROTOCOL: All patients were examined in a 3-Tesla MRI scanner (Trio, Siemens, Erlangen, Germany), and balanced steady-state free precession cine images were acquired in 2 long-axis and 7 to 9 short-axis views. ${ }^{5,6}$ After cine imaging was complete, an intravenous bolus dose of $0.025 \mathrm{mmol} / \mathrm{kg}$ gadodiamide was administered at a flow rate of $4 \mathrm{ml} / \mathrm{s}$. First-pass perfusion images were acquired with contrast injection, using a electrocardiogram-gated non-slice-selective $90^{\circ}$ saturation-recovery preparation turbo fast low angle shot pulse sequence. Three short-axis images from the apex to the base of the left ventricle were then acquired for 80 cardiac cycles during inspiratory breath-hold, yielding 80 time frames for each level at a temporal resolution of $1 \mathrm{R}$-to-R interval. Perfusion studies were performed at rest and during hyperemia induced by a 4-minute infusion of dipyridamole at a concentration of $0.14 \mathrm{mg} / \mathrm{kg}$ of body weight. After the perfusion studies, the patients received a third dose $(0.15 \mathrm{mmol} / \mathrm{kg})$ of contrast agent (cumulative dose of $0.20 \mathrm{mmol} / \mathrm{kg}$ ). Ten minutes later, late gadolinium enhancement images were acquired with the use of an inversion-recovery prepared, segmented turbo fast low angle shot pulse sequence.

IMAGE ANALYSIS: LV volume, mass, and ejection fraction were assessed using cine MRI and an automated boundary detection algorithm. The ratio of LV end-diastolic volume to LV mass was calculated and used as a measure of global end-diastolic wall stress. ${ }^{7}$ Regional end-systolic wall stress, expressed as $10^{3} \mathrm{~N} / \mathrm{m}^{2}$, was also calculated using the Janz method. ${ }^{8}$ The equation was $0.133 \times$ systolic blood pressure $\times$ the area ratio of the blood pool to ventricular wall. The perfusion index was computed from the maximal upslope of the time-intensity curves in the myocardium and normalized to the maximal upslope in the LV blood pool (Figure 1). The MPR was defined as the ratio of the upslope at stress to that at rest and was computed segment by segment. The LV myocardium at 3 short-axis levels was divided into 16 equiangular segments, with each segment assigned to a coronary artery territory, excluding the apical cap. ${ }^{9}$ These segments were defined as infarcted myocardium if the supplying coronary artery was an infarct-related artery; otherwise, they were defined as remote myocardium. To assess infarct size, we detected late gadolinium enhancement and quantified it using a signal intensity threshold +2 SDs higher than the mean signal intensity in the remote myocardium. ${ }^{10}$ Infarct size was expressed as grams of infarcted myocardium. Results of perfusion studies and late enhancement were analyzed using the Mathematica software package (Wolfram Research, Inc, Champaign, Illinois). The intraobserver variability in the perfusion results was $6 \%$ and $3 \%$ in the LV volume results.

Statistical analysis: Calculation of statistical power for the primary end point of the peak $\mathrm{VO}_{2}$, based on previous data in post-MI patients, ${ }^{11}$ showed that a power of 0.8 was needed to detect a $14 \%$ increase at a $5 \%$ significance level with a minimum of 17 subjects per group. All data are presented as the mean $\pm \mathrm{SD}$ for continuous data and as
Table 1

Baseline characteristics of postinfarction patients randomized to the nontraining or training group

\begin{tabular}{lcc}
\hline Variable & $\begin{array}{c}\text { Nontraining Group } \\
(\mathrm{n}=19)\end{array}$ & $\begin{array}{c}\text { Training Group } \\
(\mathrm{n}=20)\end{array}$ \\
\hline Age (yrs) & $52 \pm 9$ & $52 \pm 7$ \\
Body height $(\mathrm{cm})$ & $165 \pm 6$ & $167 \pm 6$ \\
Body weight $(\mathrm{kg})$ & $72 \pm 11$ & $73 \pm 10$ \\
Body mass index $\left(\mathrm{kg} / \mathrm{m}^{2}\right)$ & $26.1 \pm 3.7$ & $26.5 \pm 3.4$ \\
Body surface area $\left(\mathrm{m}^{2}\right)$ & $1.80 \pm 0.13$ & $1.81 \pm 0.12$ \\
LV ejection fraction $(\%)$ & $62 \pm 13$ & $58 \pm 12$ \\
Peak creatinine kinase (U/L) & $5,295 \pm 1937$ & $5,571 \pm 2035$ \\
Peak creatinine kinase-MB & $381 \pm 203$ & $403 \pm 223$ \\
$\quad$ (U/L) & $12(63 \%)$ & $12(60 \%)$ \\
Left anterior descending artery & & \\
$\quad$ as infarct-related artery & 0 & 0 \\
The number of vessels with & & \\
$\quad$ residual coronary stenosis & & \\
$\quad>50 \%$ & $7.9 \pm 3.5$ & $8.6 \pm 3.3$ \\
Postinfarction period (mo) & $8(42 \%)$ & $9(45 \%)$ \\
Hypertension & $3(16 \%)$ & $3(15 \%)$ \\
Diabetes mellitus & $8(42 \%)$ & $7(35 \%)$ \\
Hyperlipidemia & $7(37 \%)$ & $6(30 \%)$ \\
Remote smokers & 0 & 0 \\
Active smokers & $11(58 \%)$ & $13(65 \%)$ \\
Angiotensin-converting enzyme & & \\
$\quad$ inhibitors or ARBs use & $15(78 \%)$ & $15(75 \%)$ \\
$\beta$-blockers use & $8(42 \%)$ & $7(35 \%)$ \\
Statins use & & \\
\hline
\end{tabular}

Data are presented as the mean \pm SD or the number $(\%)$ of patients. Hypertension is defined as a repeatedly elevated blood pressure $\geq 140 / 90$ $\mathrm{mm} \mathrm{Hg}$ or current use of antihypertensive medication. Hyperlipidemia is defined as fasting total cholesterol $\geq 200 \mathrm{mg} / \mathrm{dl}$, low-density lipoprotein cholesterol $\geq 130 \mathrm{mg} / \mathrm{dl}$, triglyceride $\geq 200 \mathrm{mg} / \mathrm{dl}$, or current use of lipidlowering drugs. No significant differences were observed for any comparison between the training and nontraining groups.

$\mathrm{ARB}=$ angiotensin II receptor blockers

proportions for binary data. If the data were not distributed normally, natural logarithmic transformation was used for analysis. Correlations were tested using Pearson analysis. Baseline characteristics were compared using the unpaired Student's $t$ test for continuous data and chi-square analysis for binary data. Changes in data from the baseline to follow-up assessments were compared using the paired Student's $t$ test. A p $<0.05$ was considered statistically significant. Statistical analyses were performed using the software package SPSS, version 12.02 (SPSS, Chicago, Illinois).

\section{Results}

Clinical characteristics: Table 1 lists the demographic data for the 39 post-MI patients. There were no significant differences between the training and nontraining groups in terms of age, cardiac structure and function, infarct size, infarct duration after MI, or risk factors. Furthermore, the 2 groups did not differ in their medications. All patients maintained their own drug plan throughout the study. No patient died, was hospitalized for coronary intervention, or had worsening symptoms during the 3-month study period.

Exercise capacity and hemodynamics: Compared with the healthy controls, post-MI patients had a higher at rest 
Table 2

Cardiopulmonary function at baseline and at end of follow-up

\begin{tabular}{|c|c|c|c|c|c|}
\hline \multirow[t]{2}{*}{ Variable } & \multicolumn{2}{|c|}{ Nontraining Group $(\mathrm{n}=19)$} & \multicolumn{2}{|c|}{ Training Group $(\mathrm{n}=20)$} & \multirow[t]{2}{*}{ Healthy Controls $(\mathrm{n}=19)$} \\
\hline & Baseline & Third Month & Baseline & Third Month & \\
\hline Body mass index $\left(\mathrm{kg} / \mathrm{m}^{2}\right)$ & $26.1 \pm 3.7$ & $26.5 \pm 4.0$ & $26.5 \pm 3.4$ & $26.3 \pm 2.9$ & $25.5 \pm 2.6$ \\
\hline \multicolumn{6}{|l|}{ At rest } \\
\hline Heart rate (beats/min) & $78 \pm 14 *$ & $79 \pm 13 *$ & $77 \pm 13^{*}$ & $76 \pm 13$ & $70 \pm 8$ \\
\hline Systolic blood pressure (mm Hg) & $130 \pm 20$ & $124 \pm 19$ & $126 \pm 16$ & $122 \pm 16$ & $119 \pm 15$ \\
\hline Pressure-rate product $\left(\mathrm{mm} \mathrm{Hg} \times\right.$ beats $\left./ \mathrm{min} \times 10^{3}\right)$ & $10 \pm 3 *$ & $10 \pm 2 *$ & $10 \pm 2 *$ & $9 \pm 2$ & $8 \pm 2$ \\
\hline \multicolumn{6}{|l|}{ Peak exercise } \\
\hline Heart rate (beats/min) & $145 \pm 14$ & $143 \pm 15$ & $146 \pm 17$ & $148 \pm 15$ & $151 \pm 20$ \\
\hline Systolic blood pressure (mm Hg) & $194 \pm 32$ & $184 \pm 31$ & $182 \pm 27$ & $190 \pm 26$ & $181 \pm 26$ \\
\hline Pressure-rate product $\left(\mathrm{mm} \mathrm{Hg} \times\right.$ beats $\left./ \mathrm{min} \times 10^{3}\right)$ & $28 \pm 4$ & $26 \pm 5$ & $26 \pm 6$ & $28 \pm 5$ & $27 \pm 5$ \\
\hline Peak oxygen uptake $(\mathrm{ml} / \mathrm{kg} / \mathrm{min})$ & $22.7 \pm 3.1 *$ & $22.4 \pm 3.0^{*}$ & $22.2 \pm 3.9^{*}$ & $25.0 \pm 5.0^{\dagger}$ & $26.3 \pm 4.4$ \\
\hline Maximal workload (W) & $107 \pm 22 *$ & $105 \pm 19^{*}$ & $106 \pm 20^{*}$ & $123 \pm 21^{\dagger}$ & $134 \pm 20$ \\
\hline
\end{tabular}

Data are presented as the mean $\pm \mathrm{SD}$.

$* \mathrm{p}<0.05$ compared with healthy controls.

${ }^{\dagger} \mathrm{p}<0.05$ compared with corresponding baseline data.

Table 3

Left ventricular structure, function, wall stress, and infarct size at baseline and at end of follow-up

\begin{tabular}{|c|c|c|c|c|c|}
\hline \multirow[t]{2}{*}{ Variable } & \multicolumn{2}{|c|}{ Nontraining Group $(\mathrm{n}=19)$} & \multicolumn{2}{|c|}{ Training Group $(\mathrm{n}=20)$} & \multirow[t]{2}{*}{ Healthy Controls $(n=19)$} \\
\hline & Baseline & Third Month & Baseline & Third Month & \\
\hline Body surface area $\left(\mathrm{m}^{2}\right)$ & $1.80 \pm 0.13$ & $1.80 \pm 0.15$ & $1.81 \pm 0.12$ & $1.81 \pm 0.12$ & $1.79 \pm 0.15$ \\
\hline \multicolumn{6}{|l|}{ Structure } \\
\hline Mass index $\left(\mathrm{g} / \mathrm{m}^{2}\right)$ & $75 \pm 14^{*}$ & $77 \pm 15^{*}$ & $79 \pm 16^{*}$ & $77 \pm 15^{*}$ & $58 \pm 10$ \\
\hline End-diastolic volume index $\left(\mathrm{ml} / \mathrm{m}^{2}\right)$ & $73 \pm 19 *$ & $73 \pm 16^{*}$ & $75 \pm 18^{*}$ & $73 \pm 17 *$ & $61 \pm 12$ \\
\hline End-systolic volume index $\left(\mathrm{ml} / \mathrm{m}^{2}\right)$ & $29 \pm 14^{*}$ & $28 \pm 14 *$ & $31 \pm 14^{*}$ & $30 \pm 13^{*}$ & $16 \pm 7$ \\
\hline \multicolumn{6}{|l|}{ Function } \\
\hline Ejection fraction $(\%)$ & $62 \pm 13^{*}$ & $62 \pm 12 *$ & $58 \pm 12 *$ & $60 \pm 12 *$ & $74 \pm 7$ \\
\hline Cardiac index $\left(1 / \mathrm{min} / \mathrm{m}^{2}\right)$ & $3.0 \pm 0.6$ & $3.0 \pm 0.6$ & $3.1 \pm 0.5$ & $3.1 \pm 0.5$ & $3.1 \pm 0.5$ \\
\hline \multicolumn{6}{|l|}{ Wall stress } \\
\hline End-diastolic volume/mass ratio & $0.95 \pm 0.22$ & $0.95 \pm 0.24$ & $0.95 \pm 0.27$ & $0.97 \pm 0.25$ & $1.06 \pm 0.24$ \\
\hline \multicolumn{6}{|l|}{ End-systolic wall stress $\left(10^{3} \mathrm{~N} / \mathrm{m}^{2}\right)$} \\
\hline In remote myocardium & $6.2 \pm 2.4 *$ & $6.4 \pm 2.6^{*}$ & $6.6 \pm 2.4^{*}$ & $6.8 \pm 2.9^{*}$ & $4.4 \pm 2.8$ \\
\hline In infarcted myocardium & $14.5 \pm 9.5^{*}$ & $14.7 \pm 9.7 *$ & $14.8 \pm 9.1^{*}$ & $16.1 \pm 8.2^{*}$ & $4.4 \pm 2.8$ \\
\hline Infarct size $(\mathrm{g})$ & $22 \pm 12$ & $24 \pm 13$ & $29 \pm 15$ & $27 \pm 15$ & - \\
\hline
\end{tabular}

Data are presented as the mean $\pm \mathrm{SD}$.

$* \mathrm{p}<0.05$ compared with healthy controls.

Table 4

Myocardial perfusion index and reserve at baseline and at end of follow-up

\begin{tabular}{|c|c|c|c|c|c|}
\hline \multirow[t]{2}{*}{ Variable } & \multicolumn{2}{|c|}{ Nontraining Group $(\mathrm{n}=19)$} & \multicolumn{2}{|c|}{ Training Group $(\mathrm{n}=20)$} & \multirow[t]{2}{*}{ Healthy Controls $(\mathrm{n}=19)$} \\
\hline & Baseline & Third Month & Baseline & Third Month & \\
\hline \multicolumn{6}{|c|}{ Perfusion in remote myocardium } \\
\hline At rest perfusion index & $0.10 \pm 0.03$ & $0.11 \pm 0.04$ & $0.10 \pm 0.04$ & $0.08 \pm 0.03^{*}$ & $0.09 \pm 0.03$ \\
\hline Stress perfusion index & $0.15 \pm 0.03$ & $0.15 \pm 0.03$ & $0.15 \pm 0.05$ & $0.15 \pm 0.05$ & $0.15 \pm 0.04$ \\
\hline Perfusion reserve & $1.51 \pm 0.42^{\dagger}$ & $1.49 \pm 0.35^{\dagger}$ & $1.49 \pm 0.40^{\dagger}$ & $1.90 \pm 0.55^{*}$ & $1.78 \pm 0.37$ \\
\hline \multicolumn{6}{|c|}{ Perfusion in infarcted myocardium } \\
\hline At rest perfusion index & $0.08 \pm 0.04$ & $0.08 \pm 0.03$ & $0.08 \pm 0.03$ & $0.07 \pm 0.03^{\dagger}$ & $0.09 \pm 0.03$ \\
\hline Stress perfusion index & $0.11 \pm 0.05^{\dagger}$ & $0.11 \pm 0.05^{\dagger}$ & $0.11 \pm 0.04^{\dagger}$ & $0.12 \pm 0.05^{\dagger}$ & $0.15 \pm 0.04$ \\
\hline Perfusion reserve & $1.31 \pm 0.37^{\dagger}$ & $1.32 \pm 0.38^{\dagger}$ & $1.29 \pm 0.36^{\dagger}$ & $1.58 \pm 0.45^{*}$ & $1.78 \pm 0.37$ \\
\hline
\end{tabular}

Data are presented as the mean $\pm \mathrm{SD}$.

$* \mathrm{p}<0.05$ compared with corresponding baseline data.

${ }^{\dagger} \mathrm{p}<0.05$ compared with healthy controls.

heart rate and rate-pressure product and a lower exercise capacity (Table 2). The heart rate, blood pressure, and rate-pressure product during peak exercise were similar between patients and healthy controls. Comparing the 2 patient groups, baseline exercise capacity was similar in terms of workload and peak $\mathrm{VO}_{2}$. After training, both peak 


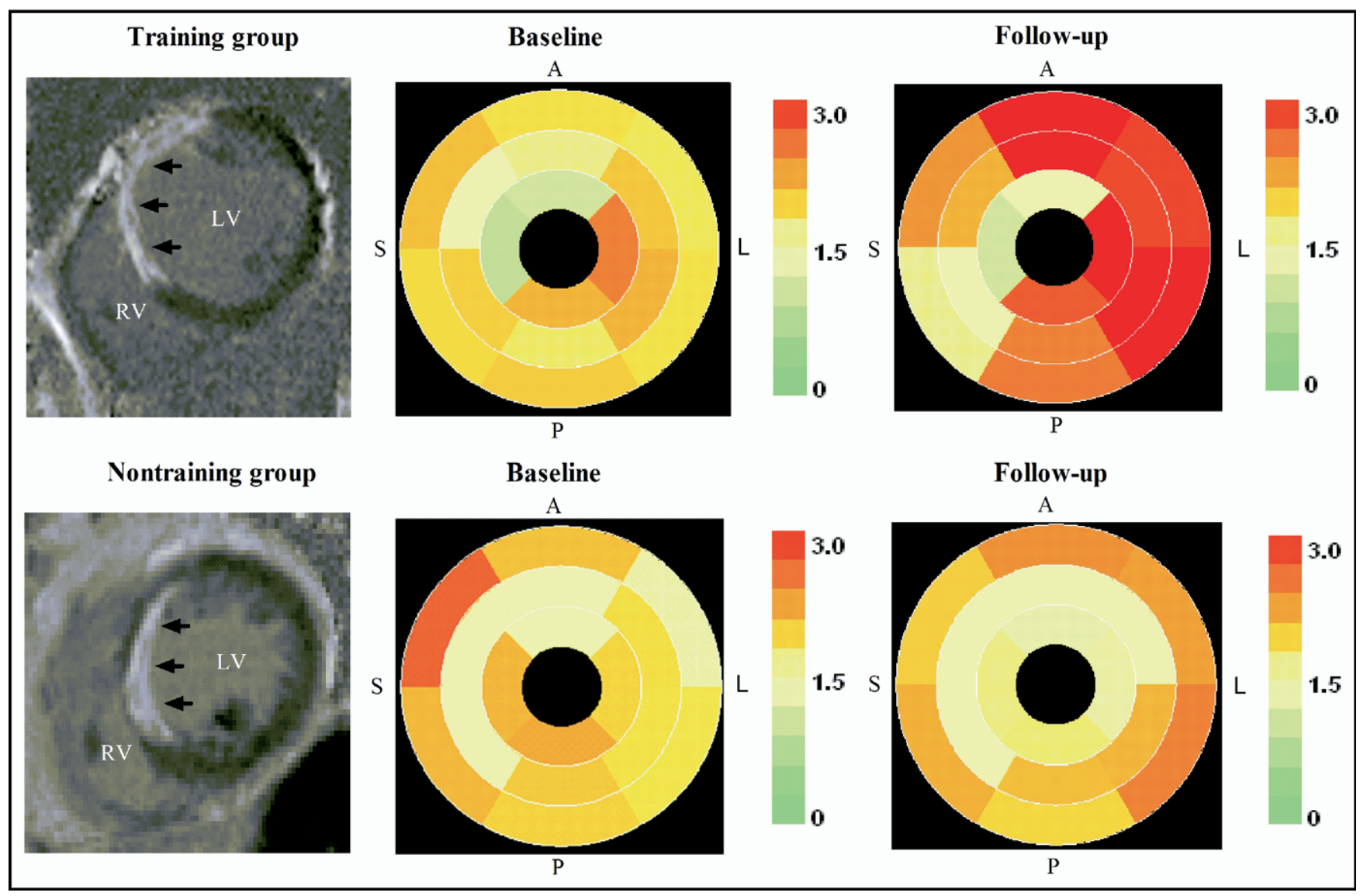

Figure 2. Bull's-eye images of MPR. A patient in the training group with an old anterior MI (top row), as shown by delayed-enhancement imaging (arrows). At baseline, low MPR can be found in the anterior and septal wall (middle panel), compatible with the infarct area shown on the delayed-enhancement imaging. After exercise training (right panel), the MPR increased, particularly in the lateral wall (remote myocardium). A patient in the nontraining group with an old anterior MI (bottom row). In this patient, the difference between baseline and follow-up was not significant. $\mathrm{A}=$ anterior; $\mathrm{L}=$ lateral; $\mathrm{P}=$ posterior; $\mathrm{RV}=$ right ventricle; $\mathrm{S}=$ septal.

$\mathrm{VO}_{2}$ and maximal workload increased by $15 \%(\mathrm{p}<0.01)$. By the third month, both parameters were at the same levels as in healthy controls. In addition, the at rest pressure-rate product, which provides a remarkably good estimate of myocardial oxygen demand, decreased to the same level as in healthy controls. In contrast, in the nontraining group, exercise capacity was unchanged after 3 months, and no changes were observed at rest and at peak exercise in heart rate, systolic blood pressure, or the rate-pressure product.

LV properties: Compared with the healthy controls, post-MI patients had lower ejection fraction, larger indices of LV mass and volume, and a higher end-systolic wall stress in the remote and infarcted myocardium (Table 3). However, there were no significant differences between post-MI patients and healthy subjects in terms of the end-diastolic volume/mass ratio, stroke volume, and cardiac index. In addition, no significant differences between baseline and follow-up levels were seen in either of the 2 patient groups in terms of LV mass, volume, end-diastolic volume/mass ratio, end-systolic wall stress, cardiac index, or infarct size.

Myocardial perfusion in the remote myocardium: At baseline, the hyperemic perfusion indices in the remote myocardium were similar in the patient groups and the healthy subjects. Post-MI patients showed a trend toward a higher at rest perfusion index, which did not reach significance, resulting in a lower MPR in the remote myocardium (Table 4). No significant differences in baseline perfusion indices and reserve in the remote myocardium were seen in the 2 patient groups. After training (Figure 2), the MPR increased by $30 \%$ from $1.49 \pm 0.40$ at baseline to $1.90 \pm$ 0.55 at the third month $(\mathrm{p}<0.01)$ to the same level as in healthy subjects $(1.90 \pm 0.55$ vs $1.78 \pm 0.37, \mathrm{p}=0.42)$. The increase in the MPR was associated with a significant decrease in the perfusion index at rest $(0.10 \pm 0.04$ before training vs $0.08 \pm 0.03$ after training, $p<0.05$ ). In the nontraining patients, the perfusion indices and reserve in the remote myocardium did not change.

Myocardial perfusion in the infarcted myocardium: In the infarcted myocardium, the perfusion index at rest in post-MI patients was similar to that in the healthy subjects, but the hyperemic perfusion index was lower, resulting in a lower MPR in the post-MI patients (Table 4). The perfusion indices and reserve in the infarcted myocardium were also lower than those in the remote myocardium. No significant differences in baseline perfusion indices and reserve in the infarcted myocardium were seen in the 2 patient groups. After training, the MPR in the infarcted myocardium in- 


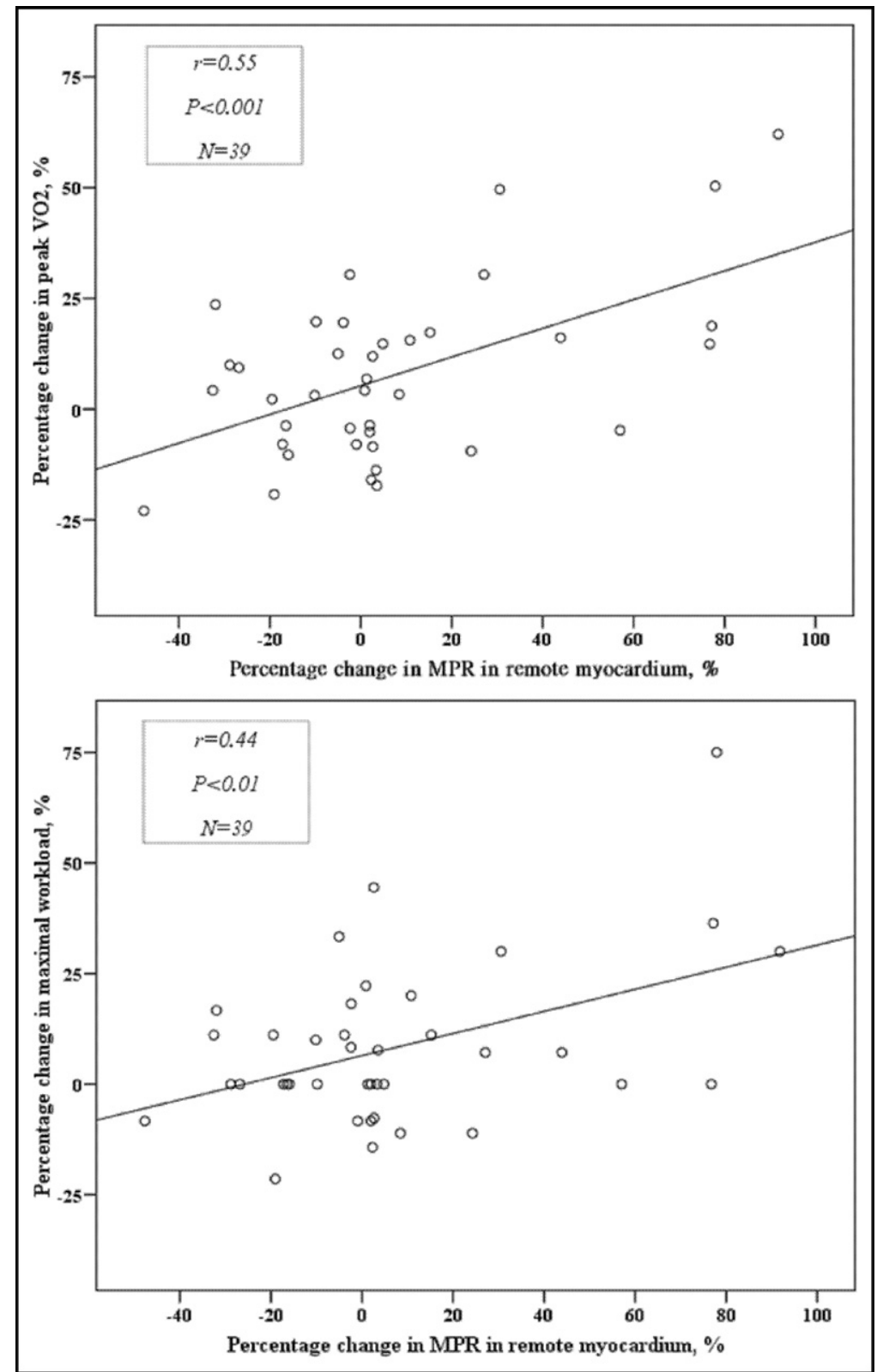

Figure 3. Relation between the percent change in the MPR in the remote myocardium and that in the peak $\mathrm{VO}_{2}$ (upper panel) or maximal work load (lower panel).

creased by $25 \%$ from $1.29 \pm 0.36$ at baseline to $1.58 \pm 0.45$ at the third month $(\mathrm{p}<0.05)$ to the same level as in healthy subjects $(1.58 \pm 0.45$ vs $1.78 \pm 0.37, \mathrm{p}=0.14$; Figure 2$)$. The increase in MPR was associated with a trend toward a lower at rest perfusion index and a higher hyperemic perfusion index. In the nontraining group, the perfusion indices and reserve did not change.

Exercise capacity and myocardial perfusion reserve: At baseline, the peak $\mathrm{VO}_{2}$ showed a significant correlation with the MPR in the remote myocardium $(r=0.30$, $\mathrm{p}=0.02)$, but not with the MPR in the infarcted myocardium $(\mathrm{r}=0.12, \mathrm{p}=0.34)$. After 3 months of training (Figure 3), a positive correlation was found between the percent change in the MPR in the remote myocardium and the percent changes in the peak $\mathrm{VO}_{2}$ and maximal workload $\left(\mathrm{r}=0.55, \mathrm{p}<0.001\right.$ for peak $\mathrm{VO}_{2} ; \mathrm{r}=0.44, \mathrm{p}<0.01$ for maximal workload). A modest, but nonsignificant, positive correlation between the percent changes in the peak $\mathrm{VO}_{2}$ and the MPR in the infarcted myocardium was also observed $(\mathrm{r}=0.35, \mathrm{p}=0.07)$.

\section{Discussion}

The present study investigated the effect of exercise-based cardiac rehabilitation on the MPR in post-MI patients. The main findings were that (1) 3 months of cardiac rehabilitation at a moderate intensity resulted in an improvement in exercise capacity to the same level as in healthy controls; (2) rehabilitation also increased MPR in both the remote and 
infarcted myocardium to the same level as in healthy controls; (3) the change in exercise capacity correlated positively with that in the MPR in the remote myocardium; and (4) LV dimension, function, wall stress, or infarct size did not change during the study period. Thus, the present findings demonstrate that the MPR in the remote myocardium may be critically involved in the beneficial effect of cardiac rehabilitation on exercise capacity.

The first finding of the present study was that, in postinfarction patients, cardiac rehabilitation improved exercise capacity to the same level as in healthy controls. Because exercise capacity is known to be an important prognostic factor in patients with cardiovascular disease, ${ }^{12}$ this suggests the survival benefit of cardiac rehabilitation and the need for cardiac rehabilitation. This study showed that the improvement in exercise capacity was accompanied by an increase in MPR during the same follow-up period, a finding similar to that reported by Hambrecht et al. ${ }^{13}$ Furthermore, our study found a close relation between exercise capacity and MPR in the remote myocardium, suggesting that the exercise-induced improvement in MPR in the remote myocardium might play an important role in improving exercise capacity.

Our present results show that the pathophysiologies of the reduced MPR in the infarcted and remote myocardium after MI are different. In the infarcted myocardium, the decreased MPR was explained by the reduced vasodilator response in the infarcted myocardium, in which the microvasculature can still be impaired, despite the successful recanalization of the epicardial artery. ${ }^{14}$ In contrast, the lower MPR in the remote myocardium was due to the higher at rest perfusion index. As detailed in Table 2, post-MI patients had a higher at rest heart rate and pressure-rate product, suggesting that post-MI patients have a higher myocardial oxygen demand at rest and thus need a greater at rest blood supply to the myocardium. Moreover, the majority of the patients (77\%) in this study were on $\beta$ blockers, allowing speculation that, if this had not been the case, both the myocardial oxygen demand and at rest perfusion index might have been higher, possibly resulting in a decreased perfusion reserve.

Cardiac rehabilitation is believed to increase the MPR, but this has not been adequately studied because of poor delineation of infarcted myocardium in the previous studies. With cardiac MRI, we showed that this beneficial effect occurred even in post-MI patients whose coronary vessels had been successfully revascularized. Interestingly, the increased MPR in the remote and infarcted myocardium after cardiac rehabilitation occurred by different mechanisms. The improvement in the MPR in the remote myocardium seen in the training group was primarily due to a decreased at rest perfusion index, and the improvement in the infarcted myocardium was due to an increased hyperemic perfusion index. In the training group, the at rest pressure-rate product, a good estimate of myocardial oxygen demand, decreased to the same level as in healthy controls after 3-month cardiac rehabilitation. This decrease might be explained by the results of a previous study ${ }^{15}$ showing that exercise training reduces myocardial oxygen demand. The decrease in myocardial oxygen demand and the at rest perfusion index resulted in an increased MPR. In contrast, we observed that the MPR in the infarcted myocardium also in- creased to the same level as in healthy controls after cardiac rehabilitation. High wall stress, an increased collagen content, and loss of resistance vessels are believed to be the main mechanisms for the decreased hyperemic perfusion index and reserve in the infarcted myocardium. ${ }^{16}$ As detailed in Table 3, wall stress and infarct size were not changed. Thus, the increase in MPR in the infarcted myocardium is probably due to recovery of function of resistant vessels after cardiac rehabilitation.

Several study limitations should be considered when interpreting these findings. First, in routine clinical practice, cardiac rehabilitation is begun as soon as possible after a cardiac event. However, studying patients soon after acute $\mathrm{MI}$ is complicated by the degree of severity and the variable rate of spontaneous improvement. ${ }^{17}$ Given our study purpose and design, only patients with stable MI were chosen. Thus, the current results may not be applicable to the cardiac population within the first 3 months after an acute MI. Second, there may have been asymptomatic restenosis of the infarct related artery in these patients. It is possible that this occurred more frequently in the control group than the training group, and this may have contributed to the differences in perfusion reserve. Because repeat coronary angiography was not performed, this cannot be evaluated in this study. Third, because only men were enrolled, further studies should be performed on women. Finally, patients were treated for 3 months only and the long-term effects of cardiac rehabilitation on these parameters remain to be established.

Acknowledgment: The authors would like to thank ChiaLin Cheng for assistance with patient enrollment and follow-up, Shu-Fang Hsiao for assistance with the cardiac rehabilitation program, and Su-Chun Huang for assistance with the MRI data.

1. Panting JR, Gatehouse PD, Yang GZ, Grothues F, Firmin DN, Collins $\mathrm{P}$, Pennell DJ. Abnormal subendocardial perfusion in cardiac syndrome $\mathrm{X}$ detected by cardiovascular magnetic resonance imaging. N Engl J Med 2002;346:1948-1953.

2. Bodi V, Sanchis J, Lopez-Lereu MP, Nunez J, Sanz R, Palau P, Gomez C, Moratal D, Chorro FJ, Llacer A. Microvascular perfusion 1 week and 6 months after myocardial infarction by first-pass perfusion cardiovascular magnetic resonance imaging. Heart 2006;92:1801-1807.

3. Lan C, Chen SY, Hsu CJ, Chiu SF, Lai JS. Improvement of cardiorespiratory function after percutaneous transluminal coronary angioplasty or coronary artery bypass grafting. Am J Phys Med Rehabil 2002;81:336-341.

4. Borg GA: Psychological basis of physical exertion. Med Sci Sports Exerc 1982;14:377-381.

5. Tseng WY, Liao TY, Wang JL. Volume and phasic function of left ventricle and left atrium in normal adults assessed with cine magnetic resonance imaging. J Cardiovasc Magn Reson 2002;4:443-457.

6. Su MY, Yang KC, Wu CC, Wu YW, Yu HY, Tseng RY, Tseng WY. First-pass myocardial perfusion cardiovascular magnetic resonance at 3 Tesla. J Cardiovasc Magn Reson 2007;9:633-644.

7. Gaasch WH. Left ventricular radius to wall thickness ratio. Am J Cardiol 1979;43:1189-1194.

8. Janz R. Estimation of local myocardial stress. Am J Physiol Heart Circ Physiol 1982;242:H875-881.

9. Cerqueira MD, Weissman NJ, Dilsizian V, Jacobs AK, Kaul S, Laskey WK, Pennell DJ, Rumberger JA, Ryan T, Verani MS; American Heart Association Writing Group on Myocardial Segmentation and Registration for Cardiac Imaging. Standardized myocardial segmentation and nomenclature for tomographic imaging of the heart: a statement 
for healthcare professionals from the Cardiac Imaging Committee of the Council on Clinical Cardiology of the American Heart Association. Int J Cardiovasc Imaging 2002;18:539-542.

10. Kim RJ, Fieno DS, Parrish TB, Harris K, Chen EL, Simonetti O, Bundy J, Finn JP, Klocke FJ, Judd RM. Relationship of MRI delayed contrast enhancement to irreversible injury, infarct age, and contractile function. Circulation 1999;100:1992-2002.

11. Taylor RS, Brown A, Ebrahim S, Jolliffe J, Noorani H, Rees K, Skidmore B, Stone JA, Thompson DR, Oldridge N. Exercise-based rehabilitation for patients with coronary heart disease: systematic review and meta-analysis of randomized controlled trials. Am J Med 2004;116:682-692.

12. Myers J, Prakash M, Froelicher V, Do D, Partington S, Atwood JE. Exercise capacity and mortality in men referred for exercise testing. N Engl J Med 2002;346:793-801.
13. Hambrecht R, Wolf A, Gielen S, Linke A, Hofer J, Erbs S, Schoene N, Schuler G. Effect of exercise on coronary endothelial function in patients with coronary artery disease. $N$ Engl J Med 2000;342:454-460.

14. Uren NG, Crake T, Lefroy DC, de Silva R, Davies GJ, Maseri A. Reduced coronary vasodilator function in infarcted and normal myocardium after myocardial infarction. $N$ Engl J Med 1994;331: 222-227.

15. Barnard RJ, MacAlpin R, Kattus AA, Buckberg GD. Effect on training on myocardial oxygen supply/demand balance. Circulation 1977;56: 289-291.

16. Camici PG, Crea F. Coronary microvascular dysfunction. $N$ Engl $J$ Med 2007;356:830-840. [Review.]

17. Kelbaek H, Eskildsen P, Hansen PF, Godtfredsen J. Spontaneous and/or training-induced haemodynamic changes after myocardial infarction. Int J Cardiol 1981;1:205-213. 